\title{
Somatotipo de los deportistas de alto rendimiento de Santiago, Chile
}

\author{
Somatotype of high performance \\ athletes of Santiago, Chile
}

\begin{abstract}
The aim of this study was to describe the somatotype of athletes from different disciplines at the High Performance Center of Santiago (CAR). Subjects and methods: We evaluated 309 male and female athletes during March 2010 to April 2011. Characteristics (mean and SD): Women: age $19.6 \pm 4.8$ years, weight $62.1 \pm$ $10.1 \mathrm{~kg}$, height, $162.7 \pm 12.5 \mathrm{~cm}$; somatotype, 4.0 to $3.7-1.9$. Male: age $21.1 \pm 5.9$ years, weight $78.8 \pm 11.6 \mathrm{~kg}$, height, 176.5 $\pm 8.3 \mathrm{~cm}$; somatotype, 3,0-5,0-2,2. Most Chilean elite athletes showed a mesomorph profile; however, there were a significant percentage of athletes who did not present this somatotype. Conclusions: This study could serve as a basis for correcting current deficiencies of Chilean athletes of high performance, being helpful for the technical team to develop a working plan for recruitment, selection and training of talents.
\end{abstract}

Key words: somatotype, athletes, Chileans.
Ximena Rodríguez P. (1)

Oscar Castillo V. (2)

Juan Tejo C. (3)

Jaime Rozowski N. (2)

(1) Programa de Magister en Nutrición, Facultad de Medicina Pontificia Universidad Católica de Chile. Santiago, Chile. (2) Unidad de Intervención Nutricional,

Departamento de Nutrición, Diabetes y Metabolismo, Facultad de Medicina, Pontificia Universidad Católica de Chile. Santiago, Chile. (3) Centro del Alto Rendimiento, Santiago de Chile.

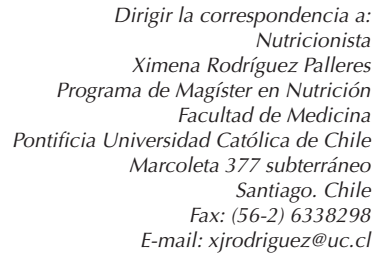

Este trabajo fue recibido el 3 de Septiembre de 2013 y aceptado para ser publicado el 18 de Enero de 2014.

\section{INTRODUCCIÓN}

El estudio del somatotipo se remonta a la antigua Grecia, donde Hipócrates y Galeno utilizaban una clasificación la cual incluía dos tipos de sujetos; los delgados y los musculosos; los primeros eran aquellos que tenían un mayor desarrollo en el eje longitudinal y normalmente tenían una personalidad introvertida, en cambio los segundos tenían un mayor desarrollo en el eje transversal y poseían una personalidad más extrovertida.

En 1940 Sheldon definió un método basado en el estudio de fotografías denominado el método fotoscópico de Sheldon, en el cual estudió a 4000 sujetos tomando tres fotografías de cada sujeto con tres planos diferentes de modo de visualizar su forma corporal, de esta manera se creó el término somatotipo para designar lo que consideraba como una entidad genética, con una cuantificación de los tres componentes primarios del cuerpo humano que son grasa, músculo y linealidad, clasificando al sujeto en endomorfo, mesomorfo y ectomorfo. Endomorfismo representa la adiposidad relativa; el mesomorfismo representa la robustez o magnitud músculoesquelética relativa y el ectomorfismo representa la linealidad relativa o delgadez de un físico (1).

En la actualidad el método de somatotipo más utilizado es el método Heath-Carter, creado en 1964, el cual utiliza la cineantropometría para la obtención del somatotipo, modifi- cando el método fotoscópico de Sheldon; demostrando que la biotipología no depende exclusivamente de la carga genética, sino también de otros factores externos como la actividad física y la nutrición, siendo modificables para conseguir el mejor rendimiento físico en el deporte practicado (2).

La combinación de los tres aspectos físicos como son endomorfo, mesomorfo y ectomorfo en una única expresión de tres números, constituye el punto fuerte del concepto del somatotipo, en donde la calificación nos dice qué tipo de físico se tiene. Entre las aplicaciones del somatotipo se utiliza para describir y comparar deportistas; caracterizar los cambios físicos durante el crecimiento, envejecimiento y el entrenamiento; y para comparar la forma relativa de hombres y mujeres.

Un mejor rendimiento deportivo no solo dependerá si las condiciones de entrenamiento físico, tanto desde el punto de vista cualitativo como cuantitativo son iguales, sino que además será en aquellos deportistas con condiciones morfológicas más favorables para la práctica del deporte en cuestión (3). En este sentido, el estudio del somatotipo cobra importancia, ya que cada especialidad deportiva presenta una serie de exigencias que obliga, en la mayoría de los casos, a poseer una determinada anatomía en los deportistas con el fin de lograr un desempeño deportivo óptimo.

Debido a la escasez de este tipo de estudios en Chile, el 
objetivo de este estudio fue describir el somatotipo mediante indicadores antropométricos de los deportistas pertenecientes a distintas disciplinas del Centro de Alto Rendimiento de Santiago, y de esta manera proveer información desde un punto de vista cineantropométrico.

\section{SUJETOS Y MÉTODO}

En este estudio descriptivo se evaluó antropométricamente a 309 deportistas de 40 disciplinas del CAR durante Marzo del 2010 hasta abril del 2011. Se trata de una muestra por conveniencia, donde el parámetro de inclusión fue que entrenaran de manera regular en el CAR.

Se midieron 124 mujeres pertenecientes a 28 deportes y 185 hombres que participaban en 33 disciplinas. En aquellos deportistas que fueron evaluados en diferentes oportunidades se consideró su última medición antropométrica.

Para la determinación del somatotipo se realizaron 12 mediciones en cada deportista: peso, estatura, pliegue tricipital, pliegue subescapular, pliegue supraespinal, pliegue pierna medial, perímetro braquial brazo relajado, perímetro braquial con brazo contraído, perímetro muslo medial, perímetro pierna media, diámetro bicondíleo de fémur y diámetro biepicondíleo de húmero. Todas las mediciones las realizó Juan Tejo según el protocolo de la Sociedad Internacional de Avances en $\mathrm{Ci}$ neantropometría (ISAK).

El peso se midió empleando una balanza SECA modelo 714 con precisión de 100 gramos (rango 0,1-130 kilos), ubicada en una superficie plana y lisa y calibrada en cero. El deportista estaba descalzo y con el mínimo de ropa. Una vez ubicado en el centro de la plataforma, se mantuvo quieto sin que su cuerpo estuviera en contacto con objetos aledaños, con el peso distribuido uniformemente en ambos pies mirando hacia el frente.

La estatura se midió con un tallímetro incorporado a la balanza SECA modelo 714 con precisión de 0,1 1 milímetros (rango $60-200 \mathrm{~cm}$ ). El sujeto se colocó de pie, descalzo con la cabeza orientada en el plano de Frankfort que une el borde interior de la órbita de los ojos y el superior del meato auditivo externo, brazos a ambos lados del tronco, extendidos y con palmas tocando cara externa de los muslos, talones juntos tocando el extremo inferior de la superficie vertical con el borde interno de los pies en el ángulo 45 a 60 grados, zona occipital, escapular, nalgas, cara posterior de las rodillas y pantorrillas tocando superficie vertical del antropómetro.

Para clasificar el estado nutricional se calculó el Índice de Masa Corporal $\left(\mathrm{IMC}=\right.$ Peso en $\mathrm{kg} /$ Talla $^{2}$ en $\mathrm{m}$ ) con la referencia OMS

Los pliegues cutáneos fueron medidos con un adipómetro Harpenden con precisión de 0.1 milímetros. Los perímetros musculares fueron medidos con una cinta métrica SECA modelo 201 metálica, flexible pero no extensible con una precisión de 0,1 cm. Los diámetros óseos se midieron con un antropómetro corto FAGA con una precisión de 0,1 cm.

Con las mediciones mencionadas se determinó el somatotipo antropométrico matemático de Health-Carter ingresándolas a las siguientes fórmulas:

Endomorfia: $-0.7182+0.1451 \times \Sigma S F-0.00068 \times \Sigma S F 2$ $+0.0000014 \times \Sigma$ SF3

$\Sigma$ SF3 = (Pliegue tricipital + pliegue subescapular + pliegue suprailiaco) $\times(170,18 /$ Estatura). Estatura en $\mathrm{cm}$. El resultado es de un número del 1 al 14.

Mesomorfia: (0.858 x Diámetro Húmero + 0.601 x Diámetro Fémur $+0.188 \times$ Perímetro Corregido del Brazo $+0.161 \times$ Perímetro Corregido de Pantorrilla) - (Estatura x 0.131) +4.5 Perímetro corregido del brazo $(\mathrm{cm})=$ Perímetro del brazo Pliegue tricipital $(\mathrm{cm})$

Perímetro corregido de la pierna $(\mathrm{cm})=$ Perímetro de la pierna - Pliegue pierna $(\mathrm{cm})$

El resultado es de un número del 1 al 14.

Ectomorfia: Su valor está entre el 0,5 y 9 y para el cálculo de la ectomorfia se debe calcular el Índice Ponderal.

Índice Ponderal = Estatura $(\mathrm{cm}) /$ Raíz cúbica del peso $(\mathrm{kg})$

Una vez obtenido el Índice Ponderal se calcula la ectormofia con los siguientes criterios:

\begin{tabular}{cc}
\hline Si IP $>40,75$ & Ectormorfia $=(I P \times 0,732)-28,58$ \\
\hline Si IP $<40,75 y>38,28$ & Ectormorfia $=($ IP $\times 0,463)-17,63$ \\
\hline Si IP $\leq 38,28$ & Ectormorfia $=0,1$
\end{tabular}

Una vez que se han calculado los tres componentes deben convertirse en x e y para elaborar la somatocarta. Dicha conversión se realiza por las siguientes formulas:

$X=$ Ectomorfia - Endomorfia

$Y=(2 \times$ Mesomorfia - (Ectomorfia + Endomorfia $)$

\section{ANÁLISIS ESTADÍSTICO}

Se presentan las variables numéricas como promedio \pm desviación estándar. Los datos se analizaron con el programa SPSS versión 15 para Windows.

\section{RESULTADOS}

La tabla 1 resume las características generales de los deportistas de sexo femenino en estudio, que fueron el tamaño

TABLA 1

Características generales (promedios \pm DE) de 124 deportistas de sexo femenino de 28 disciplinas del Centro del Alto Rendimiento Santiago de Chile.

\begin{tabular}{|c|c|c|c|c|c|c|}
\hline Disciplina & $\mathrm{n}$ & & Edad & Peso (kg) & Estatura $(\mathrm{cm})$ & IMC \\
\hline Andinismo & 3 & $\begin{array}{l}\text { Media } \\
\text { DE }\end{array}$ & $\begin{array}{c}33,6 \\
4,7\end{array}$ & $\begin{array}{c}60,7 \\
9,2\end{array}$ & $\begin{array}{c}158,3 \\
9\end{array}$ & $\begin{array}{c}24,1 \\
0.9\end{array}$ \\
\hline $\begin{array}{l}\text { Atletismo carrera } \\
\text { medio fondo }\end{array}$ & 6 & $\begin{array}{l}\text { Media } \\
\text { DE }\end{array}$ & $\begin{array}{c}19,3 \\
3,8\end{array}$ & $\begin{array}{c}54,9 \\
5,2\end{array}$ & $\begin{array}{c}163,8 \\
4,5\end{array}$ & $\begin{array}{c}20,4 \\
1,4\end{array}$ \\
\hline $\begin{array}{l}\text { Atletismo carrera } \\
\text { velocidad }\end{array}$ & 3 & $\begin{array}{l}\text { Media } \\
\text { DE }\end{array}$ & $\begin{array}{c}20,6 \\
2,3\end{array}$ & $\begin{array}{c}54,6 \\
3,4\end{array}$ & $\begin{array}{l}162,6 \\
12,3\end{array}$ & $\begin{array}{c}20,6 \\
0,7\end{array}$ \\
\hline
\end{tabular}




\begin{tabular}{|c|c|c|c|c|c|c|}
\hline $\begin{array}{l}\text { Atletismo lanzamiento } \\
\text { disco }\end{array}$ & 2 & $\begin{array}{l}\text { Media } \\
\text { DE }\end{array}$ & $\begin{array}{l}27,5 \\
0,7\end{array}$ & $\begin{array}{l}86,4 \\
14,3\end{array}$ & $\begin{array}{c}172,5 \\
3,5\end{array}$ & $\begin{array}{c}28,9 \\
3,6\end{array}$ \\
\hline $\begin{array}{l}\text { Atletismo lanzamiento } \\
\text { jabalina }\end{array}$ & 3 & $\begin{array}{l}\text { Media } \\
\text { DE }\end{array}$ & $\begin{array}{c}18,6 \\
2\end{array}$ & $\begin{array}{c}68,9 \\
8\end{array}$ & $\begin{array}{c}162,3 \\
5,1\end{array}$ & $\begin{array}{l}26,2 \\
2,5\end{array}$ \\
\hline $\begin{array}{l}\text { Atletismo } \\
\text { salto largo }\end{array}$ & 1 & $\begin{array}{l}\text { Media } \\
\text { DE }\end{array}$ & $\begin{array}{l}15 \\
-\end{array}$ & $\begin{array}{c}63 \\
-\end{array}$ & $\begin{array}{c}171 \\
-\end{array}$ & $\begin{array}{c}21,5 \\
-\end{array}$ \\
\hline Atletismo valla & 1 & $\begin{array}{l}\text { Media } \\
\text { DE }\end{array}$ & $\begin{array}{c}21 \\
-\end{array}$ & $\begin{array}{c}52,1 \\
-\end{array}$ & $\begin{array}{c}163 \\
-\end{array}$ & $\begin{array}{c}19,6 \\
-\end{array}$ \\
\hline Basquetball & 10 & $\begin{array}{l}\text { Media } \\
\text { DE }\end{array}$ & $\begin{array}{c}18,5 \\
5,2\end{array}$ & $\begin{array}{c}68,9 \\
6,5\end{array}$ & $\begin{array}{c}174,1 \\
3,9\end{array}$ & $\begin{array}{c}20,9 \\
5,7\end{array}$ \\
\hline Bicicross & 3 & $\begin{array}{l}\text { Media } \\
\text { DE }\end{array}$ & $\begin{array}{c}16,6 \\
2,8\end{array}$ & $\begin{array}{c}58,4 \\
1,4\end{array}$ & $\begin{array}{c}155,6 \\
7,3\end{array}$ & $\begin{array}{c}24,1 \\
1,7\end{array}$ \\
\hline Bowling & 2 & $\begin{array}{l}\text { Media } \\
\text { DE }\end{array}$ & $\begin{array}{l}21 \\
1,4\end{array}$ & $\begin{array}{c}80 \\
24,4\end{array}$ & $\begin{array}{l}164,5 \\
10,6\end{array}$ & $\begin{array}{c}29,2 \\
5,2\end{array}$ \\
\hline Canotaje & 1 & $\begin{array}{l}\text { Media } \\
\text { DE }\end{array}$ & $\begin{array}{l}19 \\
-\end{array}$ & $\begin{array}{c}54,4 \\
-\end{array}$ & $\begin{array}{c}155 \\
-\end{array}$ & $\begin{array}{c}22,6 \\
-\end{array}$ \\
\hline Ciclismo & 7 & $\begin{array}{l}\text { Media } \\
\text { DE }\end{array}$ & $\begin{array}{c}19,5 \\
4\end{array}$ & $\begin{array}{c}56,5 \\
7,6\end{array}$ & $\begin{array}{c}185,7 \\
5,3\end{array}$ & $\begin{array}{c}22,4 \\
3,1\end{array}$ \\
\hline Esgrima & 4 & $\begin{array}{l}\text { Media } \\
\text { DE }\end{array}$ & $\begin{array}{c}18,7 \\
3,5\end{array}$ & $\begin{array}{c}58,6 \\
7\end{array}$ & $\begin{array}{l}163 \\
4,6\end{array}$ & $\begin{array}{l}22 \\
1,9\end{array}$ \\
\hline Gimnasia artística & 4 & $\begin{array}{l}\text { Media } \\
\text { DE }\end{array}$ & $\begin{array}{c}23,7 \\
5,5\end{array}$ & $\begin{array}{c}53,7 \\
8,1\end{array}$ & $\begin{array}{c}155,7 \\
7,8\end{array}$ & $\begin{array}{l}22 \\
1,8\end{array}$ \\
\hline Handball & 25 & $\begin{array}{l}\text { Media } \\
\text { DE }\end{array}$ & $\begin{array}{c}19,4 \\
3,9\end{array}$ & $\begin{array}{l}68,1 \\
11,1\end{array}$ & $\begin{array}{c}167,7 \\
7\end{array}$ & $\begin{array}{c}24,1 \\
3,2\end{array}$ \\
\hline Judo & 3 & $\begin{array}{l}\text { Media } \\
\text { DE }\end{array}$ & $\begin{array}{l}17,6 \\
2,5\end{array}$ & $\begin{array}{c}58,8 \\
7,8\end{array}$ & $\begin{array}{l}153 \\
3,4\end{array}$ & $\begin{array}{c}25,1 \\
2,5\end{array}$ \\
\hline Karate & 2 & $\begin{array}{c}\text { Media } \\
\text { DE }\end{array}$ & $\begin{array}{c}27,5 \\
2,1\end{array}$ & $\begin{array}{c}60,8 \\
0,4\end{array}$ & $\begin{array}{c}161 \\
1,4\end{array}$ & $\begin{array}{c}23,5 \\
0,5\end{array}$ \\
\hline Levantamiento pesa & 2 & $\begin{array}{c}\text { Media } \\
\text { DE }\end{array}$ & $\begin{array}{l}22 \\
2,8\end{array}$ & $\begin{array}{c}55,5 \\
1,2\end{array}$ & $\begin{array}{l}152 \\
2,8\end{array}$ & $\begin{array}{l}24 \\
0,3\end{array}$ \\
\hline Mountainbike & 2 & $\begin{array}{c}\text { Media } \\
\text { DE }\end{array}$ & $\begin{array}{c}18,5 \\
0,7\end{array}$ & $\begin{array}{c}57,2 \\
0,1\end{array}$ & $\begin{array}{c}164,5 \\
2,1\end{array}$ & $\begin{array}{c}21,1 \\
0,6\end{array}$ \\
\hline Nado sincronizado & 1 & $\begin{array}{c}\text { Media } \\
\text { DE }\end{array}$ & $\begin{array}{c}17 \\
-\end{array}$ & $\begin{array}{c}58,5 \\
-\end{array}$ & $\begin{array}{c}158 \\
-\end{array}$ & $\begin{array}{c}23,4 \\
-\end{array}$ \\
\hline Patinaje artístico & 1 & $\begin{array}{l}\text { Media } \\
\text { DE }\end{array}$ & $\begin{array}{c}14 \\
-\end{array}$ & $\begin{array}{c}74,1 \\
-\end{array}$ & $\begin{array}{c}164 \\
-\end{array}$ & $\begin{array}{c}27,6 \\
-\end{array}$ \\
\hline Patín carrerea & 23 & $\begin{array}{l}\text { Media } \\
\text { DE }\end{array}$ & $\begin{array}{c}17,7 \\
4,2\end{array}$ & $\begin{array}{c}58,8 \\
7,3\end{array}$ & $\begin{array}{c}161,9 \\
6\end{array}$ & $\begin{array}{c}22,3 \\
1,9\end{array}$ \\
\hline Salto ornamental & 3 & $\begin{array}{l}\text { Media } \\
\text { DE }\end{array}$ & $\begin{array}{l}16 \\
2,6\end{array}$ & $\begin{array}{c}52,6 \\
1,7\end{array}$ & $\begin{array}{l}158 \\
5,5\end{array}$ & $\begin{array}{c}21,1 \\
1,1\end{array}$ \\
\hline Taekwondo & 4 & $\begin{array}{c}\text { Media } \\
\text { DE }\end{array}$ & $\begin{array}{l}25 \\
4,2\end{array}$ & $\begin{array}{c}60,9 \\
6,6\end{array}$ & $\begin{array}{l}166 \\
7,2\end{array}$ & $\begin{array}{l}22 \\
0,8\end{array}$ \\
\hline Tenis de mesa & 5 & $\begin{array}{c}\text { Media } \\
\text { DE }\end{array}$ & $\begin{array}{c}17,4 \\
4,8\end{array}$ & $\begin{array}{c}57,9 \\
6,3\end{array}$ & $\begin{array}{c}161,2 \\
6,4\end{array}$ & $\begin{array}{c}22,2 \\
1,4\end{array}$ \\
\hline Tiro al arco & 1 & $\begin{array}{l}\text { Media } \\
\text { DE }\end{array}$ & $\begin{array}{c}16 \\
-\end{array}$ & $\begin{array}{c}73,1 \\
-\end{array}$ & $\begin{array}{c}169 \\
-\end{array}$ & $\begin{array}{c}25,6 \\
-\end{array}$ \\
\hline Tiro al vuelo & 1 & $\begin{array}{l}\text { Media } \\
\text { DE }\end{array}$ & $\begin{array}{c}20 \\
-\end{array}$ & $\begin{array}{c}54,8 \\
-\end{array}$ & $\begin{array}{c}160 \\
-\end{array}$ & $\begin{array}{c}21,4 \\
-\end{array}$ \\
\hline Voleyball playa & 1 & $\begin{array}{c}\text { Media } \\
\text { DE }\end{array}$ & $\begin{array}{c}23 \\
-\end{array}$ & $\begin{array}{c}72,6 \\
-\end{array}$ & $\begin{array}{c}173 \\
-\end{array}$ & $\begin{array}{c}24,3 \\
-\end{array}$ \\
\hline Total & 124 & $\begin{array}{c}\text { Media } \\
\text { DE }\end{array}$ & $\begin{array}{c}19,6 \\
4,8\end{array}$ & $\begin{array}{l}62,1 \\
10,1\end{array}$ & $\begin{array}{c}162,7 \\
12,5\end{array}$ & $\begin{array}{c}22,9 \\
3,1\end{array}$ \\
\hline
\end{tabular}


de la muestra, edad, peso, estatura e IMC. Dichas deportistas tenían un promedio de edad de 19 años con 6 meses, cuyo peso estaba entorno a los 62 kilos y la estatura en alrededor de $162 \mathrm{~cm}$. El promedio de IMC fue de 22,9 clasificándose como estado nutricional normal. En cuanto a la edad, la deportista de patinaje artístico presentó la menor edad, en cambio la mayor edad fue de las deportistas de andinismo. Sobre el peso, las deportistas de salto ornamental tuvieron el menor peso, a diferencia de lanzamiento del disco cuyas deportistas obtuvieron el mayor peso. Las deportistas de levantamiento de pesa presentaron la menor estatura y las más altas fueron las deportistas de ciclismo. Se destaca que el valor mínimo de IMC es corresponde a atletismo valla y el máximo pertenece a bowling.

La tabla 2 muestra las características generales de los deportistas de sexo masculino de este estudio, que fueron las mismas que los deportistas de sexo femenino. La edad de los deportistas fue de alrededor de los 21 años, con un peso y estatura promedio de 75,8 kilos y $176,6 \mathrm{~cm}$ respectivamente. En cuanto al estado nutricional se clasificó como normal. Los nadadores presentaron la menor edad, a diferencia de los andinistas que presentaron la mayor edad. Se destaca que el menor peso corresponde al deportista de salto ornamental y el máximo pertenece al lanzamiento del martillo. Sobre la estatura el deportista de salto ornamental obtuvo la menor estatura, en cambio los voleibolistas indoor fueron los más altos. El menor IMC correspondió a los atletas de carrera de medio fondo y el mayor al deportista de lanzamiento del martillo.

La somatocarta de los deportistas de sexo femenino se muestra en la figura 1 y 2 en donde se grafican las 26 disciplinas que practicaban las deportistas de este estudio.

Para el caso de los deportistas de sexo masculino la somatocarta se grafica en la figura 3 y 4 con los 32 deportes que practicaban los deportistas en este estudio.

\section{DISCUSIÓN}

En este estudio se describe el somatotipo de las de las distintas disciplinas del Centro de Alto Rendimiento de Santiago de Chile, siendo comparado con los somatotipos reportados en otros estudios.

\section{Deportistas de sexo masculino}

Los deportistas de salto largo, lanzamiento de bala, lanzamiento de martillo, jabalina, bicicross, bowling, buceo, canotaje, mountainbike, patinaje artístico, remo y salto ornamental, obtuvieron un somatotipo predominantemente mesomórfico, lo que se traduce en sujetos con bajo contenido graso para su estatura, gran cantidad de tejido muscular y desarrollo óseo para su estatura y una figura general en donde la linealidad de su cuerpo es relativamente baja.

En el esquí acuático el somatotipo que predominó fue el ectomórfico, lo que indica una linealidad relativa, vale decir predominio de la linealidad sobre la masa muscular asociado a bajo porcentaje de masa grasa y poco desarrollo de la masa muscular. Caso contrario es el grupo de andinistas en donde el somatotipo predominante fue el endomórfico el que representa un alto contenido de grasa para su estatura.

En el caso de los karatecas, judocas, esgrimistas, boxeadores, gimnastas, ciclistas y nadadores, ellos obtuvieron un perfil predominantemente mesomórfico, el cual coincide con deportistas de élite para dichas disciplinas. Este somatotipo presenta un nivel medio de grasa y predominio de la masa muscular, lo que favorece a la performance de estos atletas (4-15).

En las disciplinas que se practican en patines como son hockey patín y patín carrera, los deportistas chilenos presentaron un somatotipo mesomorfo, somatotipo que fue reportado en otros estudios realizados en deportistas de alto rendimiento $(16,17)$.

En los deportes en donde la composición corporal cobra mayor relevancia por ser deportes de contacto físico con sus

TABLA 2

Características generales (promedios \pm DE) de 185 deportistas de sexo masculino de 33 disciplinas del Centro del Alto Rendimiento, Santiago de Chile.

\begin{tabular}{|c|c|c|c|c|c|c|}
\hline Disciplina & $\mathrm{n}$ & & Edad & Peso (kg) & Estatura $(\mathrm{cm})$ & $I M C$ \\
\hline Andinismo & 4 & $\begin{array}{l}\text { Media } \\
\text { DE }\end{array}$ & $\begin{array}{c}30,5 \\
3,8\end{array}$ & $\begin{array}{l}77,5 \\
8,3\end{array}$ & $\begin{array}{c}173,5 \\
2,8\end{array}$ & $\begin{array}{c}25,7 \\
2,2\end{array}$ \\
\hline $\begin{array}{l}\text { Atletismo carrera } \\
\text { medio fondo }\end{array}$ & 8 & $\begin{array}{l}\text { Media } \\
\text { DE }\end{array}$ & $\begin{array}{c}20,3 \\
3,9\end{array}$ & $\begin{array}{c}67,6 \\
3,8\end{array}$ & $\begin{array}{c}180,2 \\
5\end{array}$ & $\begin{array}{c}20,8 \\
0,4\end{array}$ \\
\hline $\begin{array}{l}\text { Atletismo carrera } \\
\text { velocidad }\end{array}$ & 6 & $\begin{array}{l}\text { Media } \\
\text { DE }\end{array}$ & $\begin{array}{l}23 \\
7,3\end{array}$ & $\begin{array}{l}73,4 \\
11,9\end{array}$ & $\begin{array}{c}175,5 \\
5,2\end{array}$ & $\begin{array}{c}23,8 \\
3,3\end{array}$ \\
\hline $\begin{array}{l}\text { Atletismo } \\
\text { lanzamiento bala }\end{array}$ & 1 & $\begin{array}{l}\text { Media } \\
\text { DE }\end{array}$ & $\begin{array}{l}19 \\
-\end{array}$ & $\begin{array}{c}99,7 \\
-\end{array}$ & $\begin{array}{c}184 \\
-\end{array}$ & $\begin{array}{c}29,5 \\
-\end{array}$ \\
\hline $\begin{array}{l}\text { Atletismo lanzamiento } \\
\text { jabalina }\end{array}$ & 1 & $\begin{array}{l}\text { Media } \\
\text { DE }\end{array}$ & $\begin{array}{l}18 \\
-\end{array}$ & $\begin{array}{l}81 \\
-\end{array}$ & $\begin{array}{c}184 \\
-\end{array}$ & $\begin{array}{c}23,9 \\
-\end{array}$ \\
\hline $\begin{array}{l}\text { Atletismo } \\
\text { lanzamiento martillo }\end{array}$ & 1 & $\begin{array}{l}\text { Media } \\
\text { DE }\end{array}$ & 23 & $\begin{array}{c}111,7 \\
-\end{array}$ & $\begin{array}{c}179 \\
-\end{array}$ & $\begin{array}{c}34,9 \\
-\end{array}$ \\
\hline $\begin{array}{l}\text { Atletismo } \\
\text { salto largo }\end{array}$ & 1 & $\begin{array}{l}\text { Media } \\
\text { DE }\end{array}$ & $\begin{array}{c}24 \\
-\end{array}$ & $\begin{array}{c}76,5 \\
-\end{array}$ & $\begin{array}{c}181 \\
-\end{array}$ & $\begin{array}{c}23,4 \\
-\end{array}$ \\
\hline Bicicross & 7 & $\begin{array}{c}\text { Media } \\
\text { DE }\end{array}$ & $\begin{array}{l}18 \\
2,1\end{array}$ & $\begin{array}{l}76,6 \\
12,3\end{array}$ & $\begin{array}{c}175,4 \\
4,9\end{array}$ & $\begin{array}{c}24,9 \\
4\end{array}$ \\
\hline
\end{tabular}




\begin{tabular}{|c|c|c|c|c|c|c|}
\hline Boxeo & 8 & $\begin{array}{c}\text { Media } \\
\text { DE }\end{array}$ & $\begin{array}{l}18 \\
1,5\end{array}$ & $\begin{array}{c}69,2 \\
9,1\end{array}$ & $\begin{array}{c}173,3 \\
6,8\end{array}$ & $\begin{array}{l}23 \\
1,8\end{array}$ \\
\hline Buceo & 1 & $\begin{array}{c}\text { Media } \\
\text { DE }\end{array}$ & $\begin{array}{c}32 \\
-\end{array}$ & $\begin{array}{c}72,9 \\
-\end{array}$ & $\begin{array}{c}170 \\
-\end{array}$ & $\begin{array}{c}25,2 \\
-\end{array}$ \\
\hline Ciclismo & 6 & $\begin{array}{c}\text { Media } \\
\text { DE }\end{array}$ & $\begin{array}{c}20,8 \\
7,4\end{array}$ & $\begin{array}{l}74,7 \\
15,5\end{array}$ & $\begin{array}{c}174,3 \\
5,9\end{array}$ & $\begin{array}{c}24,5 \\
4,1\end{array}$ \\
\hline Esgrima & 8 & $\begin{array}{c}\text { Media } \\
\text { DE }\end{array}$ & $\begin{array}{c}25,5 \\
6,3\end{array}$ & $\begin{array}{c}79,5 \\
7,6\end{array}$ & $\begin{array}{c}175,2 \\
8\end{array}$ & $\begin{array}{c}25,8 \\
1\end{array}$ \\
\hline Handball & 18 & $\begin{array}{c}\text { Media } \\
\text { DE }\end{array}$ & $\begin{array}{c}21,1 \\
3,1\end{array}$ & $\begin{array}{c}80 \\
11,4\end{array}$ & $\begin{array}{c}181,1 \\
7,7\end{array}$ & $\begin{array}{c}24,3 \\
2,2\end{array}$ \\
\hline Hockey patín & 13 & $\begin{array}{c}\text { Media } \\
\text { DE }\end{array}$ & $\begin{array}{c}18,5 \\
2,7\end{array}$ & $\begin{array}{l}77,1 \\
8,2\end{array}$ & $\begin{array}{c}176 \\
6\end{array}$ & $\begin{array}{c}24,8 \\
2\end{array}$ \\
\hline Judo & 4 & $\begin{array}{c}\text { Media } \\
\text { DE }\end{array}$ & $\begin{array}{c}18,7 \\
2,2\end{array}$ & $\begin{array}{c}82,6 \\
4,8\end{array}$ & $\begin{array}{c}178,2 \\
6,8\end{array}$ & $\begin{array}{l}26 \\
1,9\end{array}$ \\
\hline Karate & 3 & $\begin{array}{c}\text { Media } \\
\text { DE }\end{array}$ & $\begin{array}{c}21,6 \\
1,5\end{array}$ & $\begin{array}{c}78,8 \\
14\end{array}$ & $\begin{array}{c}175,3 \\
10,7\end{array}$ & $\begin{array}{c}25,4 \\
1,8\end{array}$ \\
\hline Levantamiento pesa & 7 & $\begin{array}{c}\text { Media } \\
\text { DE }\end{array}$ & $\begin{array}{c}21,1 \\
3,1\end{array}$ & $\begin{array}{l}81,8 \\
17,2\end{array}$ & $\begin{array}{c}170,8 \\
8,7\end{array}$ & $\begin{array}{r}27,9 \\
4,6\end{array}$ \\
\hline Patín carrera & 17 & $\begin{array}{c}\text { Media } \\
\text { DE }\end{array}$ & $\begin{array}{c}18,4 \\
2,6\end{array}$ & $\begin{array}{c}70,5 \\
8,3\end{array}$ & $\begin{array}{c}172,8 \\
7\end{array}$ & $\begin{array}{c}23,4 \\
1,9\end{array}$ \\
\hline Patinaje artístico & 1 & $\begin{array}{c}\text { Media } \\
\text { DE }\end{array}$ & $\begin{array}{l}19 \\
-\end{array}$ & $\begin{array}{c}76,4 \\
-\end{array}$ & $\begin{array}{c}175 \\
-\end{array}$ & $\begin{array}{c}25 \\
-\end{array}$ \\
\hline Remo & 1 & $\begin{array}{c}\text { Media } \\
\text { DE }\end{array}$ & $\begin{array}{c}20 \\
-\end{array}$ & $\begin{array}{c}81,2 \\
-\end{array}$ & $\begin{array}{c}181 \\
-\end{array}$ & $\begin{array}{c}24,8 \\
-\end{array}$ \\
\hline Rugby & 14 & $\begin{array}{c}\text { Media } \\
\text { DE }\end{array}$ & $\begin{array}{c}23,5 \\
2,8\end{array}$ & $\begin{array}{l}85 \\
5,3\end{array}$ & $\begin{array}{c}178,4 \\
5,9\end{array}$ & $\begin{array}{c}26,7 \\
1,4\end{array}$ \\
\hline Salto ornamental & 1 & $\begin{array}{c}\text { Media } \\
\text { DE }\end{array}$ & $\begin{array}{c}17 \\
-\end{array}$ & $\begin{array}{c}57,9 \\
-\end{array}$ & $\begin{array}{c}165 \\
-\end{array}$ & $\begin{array}{c}21,3 \\
-\end{array}$ \\
\hline Taikwondo & 5 & $\begin{array}{c}\text { Media } \\
\text { DE }\end{array}$ & $\begin{array}{c}22,6 \\
3,8\end{array}$ & $\begin{array}{l}69,6 \\
14,2\end{array}$ & $\begin{array}{l}173 \\
9,7\end{array}$ & $\begin{array}{l}23 \\
2,4\end{array}$ \\
\hline Tenis de mesa & 6 & $\begin{array}{c}\text { Media } \\
\text { DE }\end{array}$ & $\begin{array}{c}28 \\
15,5\end{array}$ & $\begin{array}{l}71,7 \\
12,1\end{array}$ & $\begin{array}{c}166,3 \\
13\end{array}$ & $\begin{array}{l}26 \\
4,3\end{array}$ \\
\hline Voleyball indoor & 14 & $\begin{array}{c}\text { Media } \\
\text { DE }\end{array}$ & $\begin{array}{c}18,7 \\
5\end{array}$ & $\begin{array}{l}78,1 \\
11,1\end{array}$ & $\begin{array}{c}188,9 \\
7,6\end{array}$ & $\begin{array}{c}21,8 \\
2,7\end{array}$ \\
\hline Voleyball playa & 1 & $\begin{array}{c}\text { Media } \\
\text { DE }\end{array}$ & $\begin{array}{l}21 \\
-\end{array}$ & $\begin{array}{c}81,1 \\
-\end{array}$ & $\begin{array}{c}169 \\
-\end{array}$ & $\begin{array}{c}22 \\
-\end{array}$ \\
\hline Total & 185 & $\begin{array}{c}\text { Media } \\
\text { DE }\end{array}$ & $\begin{array}{c}21,1 \\
5,9\end{array}$ & $\begin{array}{l}75,8 \\
11,6\end{array}$ & $\begin{array}{c}176,5 \\
8,3\end{array}$ & $\begin{array}{c}24,2 \\
3,0\end{array}$ \\
\hline
\end{tabular}


Somatocarta de los deportistas de sexo femenino de las disciplinas 1 a la 17 del Centro de Alto Rendimiento, Santiago de Chile.

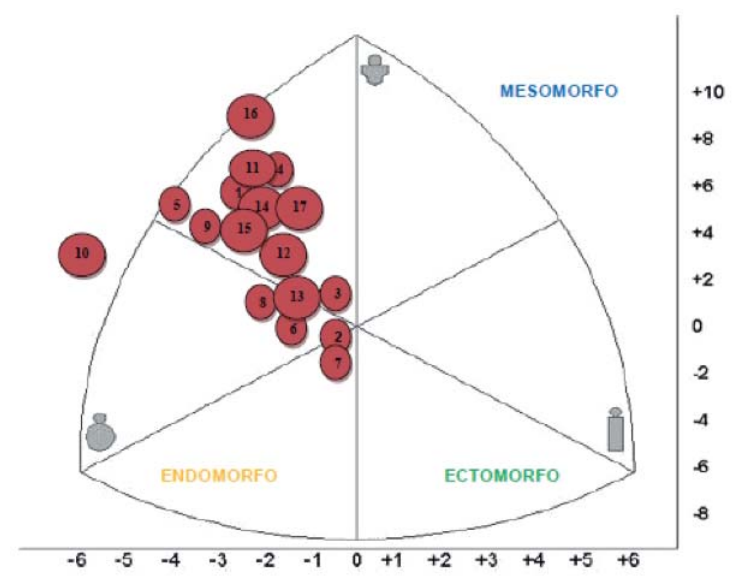

\begin{tabular}{|c|c|c|c|c|c|c|c|}
\hline $\mathbf{N}^{\circ}$ & Disciplina & & Endomorfo & Mesomorfo & Ectomorfo & $\mathbf{X}$ & $\mathbf{Y}$ \\
\hline \multirow[t]{2}{*}{1} & \multirow{2}{*}{ Andinismo } & Media & 3,7 & 5,3 & 1,1 & $-3,0$ & 5,7 \\
\hline & & DE & 0,4 & 0,2 & 0,1 & & \\
\hline \multirow[t]{2}{*}{2} & \multirow{2}{*}{ Atletismo carrera medio fondo } & Media & 2,9 & 3,3 & 3,0 & 0 & $-5,2$ \\
\hline & & $\mathrm{DE}$ & 0,9 & 1,0 & 0,7 & & \\
\hline \multirow[t]{2}{*}{3} & \multirow{2}{*}{ Atletismo carrera velocidad } & Media & 3,1 & 0,3 & 2,8 & 0,1 & $-0,3$ \\
\hline & & $\mathrm{DE}$ & 0,2 & 6,0 & 0,2 & & \\
\hline \multirow[t]{2}{*}{4} & \multirow{2}{*}{ Atletismo lanzamiento disco } & Media & 4,7 & 5,9 & 0,5 & $-4,2$ & 6,5 \\
\hline & & $\mathrm{DE}$ & 1,0 & 0,2 & 0,6 & & \\
\hline \multirow[t]{2}{*}{5} & \multirow{2}{*}{ Atletismo lanzamiento Jabalina } & Media & 4,4 & 6,3 & 0,7 & $-3,6$ & 7,2 \\
\hline & & DE & 1,2 & 0,6 & 0,7 & & \\
\hline \multirow[t]{2}{*}{6} & \multirow{2}{*}{ Atletismo salto largo } & Media & 4,2 & 3,6 & 2,9 & $-1,2$ & 0 \\
\hline & & $\mathrm{DE}$ & - & - & - & & \\
\hline \multirow[t]{2}{*}{7} & \multirow{2}{*}{ Atletismo valla } & Media & 3,5 & 3,0 & 3,4 & $-0,1$ & $-0,8$ \\
\hline & & DE & - & - & - & & \\
\hline \multirow[t]{2}{*}{8} & \multirow{2}{*}{ Basquetball } & Media & 4,3 & 0,3 & 2,5 & $-2,2$ & $-8,3$ \\
\hline & & DE & 0,7 & 5,5 & 0,9 & & \\
\hline \multirow[t]{2}{*}{9} & \multirow{2}{*}{ Bicicross } & Media & 4,4 & 2,1 & 1,1 & $-3,2$ & $-1,3$ \\
\hline & & $\mathrm{DE}$ & 1,1 & 5,9 & 0,8 & & \\
\hline \multirow[t]{2}{*}{10} & \multirow{2}{*}{ Bowling } & Media & 5,1 & 7,7 & 0,5 & $-6,6$ & 3,5 \\
\hline & & $\mathrm{DE}$ & 1,3 & 2,0 & 0,2 & & \\
\hline \multirow[t]{2}{*}{11} & \multirow{2}{*}{ Canotaje } & Media & 3,1 & 6,2 & 1,4 & $-1,7$ & 7,8 \\
\hline & & DE & - & - & - & & \\
\hline \multirow[t]{2}{*}{12} & \multirow{2}{*}{ Ciclismo } & Media & 3,8 & 1,7 & 1,9 & $-1,3$ & $-0,09$ \\
\hline & & $\mathrm{DE}$ & 1,8 & 4,7 & 1,5 & & \\
\hline \multirow[t]{2}{*}{13} & \multirow{2}{*}{ Esgrima } & Media & 3,9 & 3,4 & 2,4 & $-1,5$ & 1 \\
\hline & & DE & 1,0 & 0,8 & 0,7 & & \\
\hline \multirow[t]{2}{*}{14} & \multirow{2}{*}{ Gimnasia artística } & Media & 3,0 & 2,6 & 1,9 & $-1,3$ & 4,3 \\
\hline & & $\mathrm{DE}$ & 1,1 & 4,6 & 0,8 & & \\
\hline 15 & Handhall & Media & 4,7 & 4,9 & 1,7 & $-2,9$ & 2,4 \\
\hline & Handoall & $\mathrm{DE}$ & 1,3 & 1,3 & 1,2 & & \\
\hline 16 & Judo & Media & 3,6 & 6,5 & 0,7 & $-2,8$ & 8,6 \\
\hline & & $\mathrm{DE}$ & 1,3 & 0,5 & 0,3 & & \\
\hline 17 & Karate & Media & 3,1 & 5,2 & 1,4 & $-1,6$ & 5,8 \\
\hline & Karate & $\mathrm{DE}$ & 0,2 & 0,07 & 0,3 & & \\
\hline
\end{tabular}


contrincantes como son el rugby y la lucha libre, o en el caso del levantamiento de pesa en donde se tiene que levantar cargas de gran peso, los atletas de estas disciplinas que participaron en este estudio presentaron un mayor predominio del desarrollo músculo-esquelético, siendo similares a estudios llevados a cabo en deportistas de olimpiadas. Este elevado valor del componente mesomorfo se correlaciona con la necesidad de un desarrollo muscular importante necesario para enfrentar a los contrincantes (13,18-21).

En las disciplinas de tenis de mesa, handball, taekwondo, atletas de carrera de medio fondo y velocidad los somatotipos encontrados en este estudio no coinciden con los reportados

\section{FIGURA 2}

Somatocarta de los deportistas de sexo femenino de las disciplinas 18 a la 28 del Centro de Alto Rendimiento, Santiago de Chile.

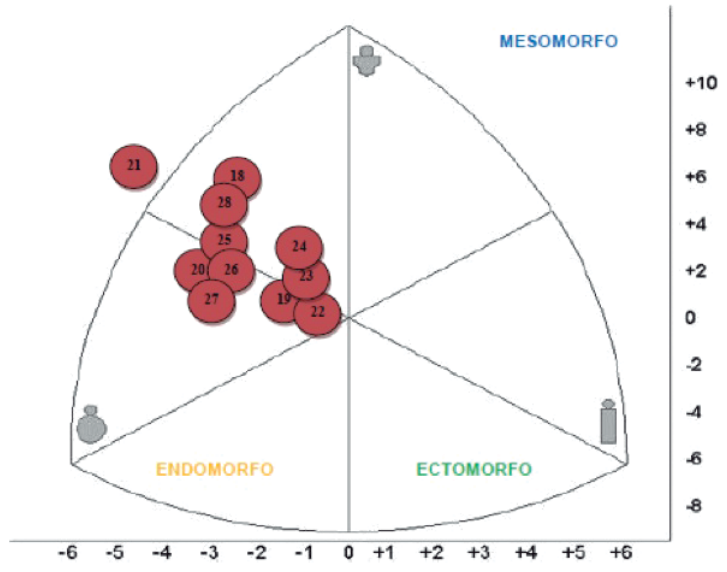

\begin{tabular}{|c|c|c|c|c|c|c|c|}
\hline $\mathbf{N}^{\circ}$ & Disciplina & & Endomorfo & Mesomorfo & Ectomorfo & $\mathbf{X}$ & $\mathbf{Y}$ \\
\hline \multirow[t]{2}{*}{18} & \multirow{2}{*}{ Levantamiento pesa } & Media & 3,2 & 5,8 & 0,8 & $-2,3$ & 7,5 \\
\hline & & DE & 0,5 & 0,8 & 0,2 & & \\
\hline \multirow[t]{2}{*}{19} & \multirow{2}{*}{ Mountainbike } & Media & 3,6 & 3,8 & 2,7 & $-0,9$ & 1,3 \\
\hline & & $\mathrm{DE}$ & 2 & 0,1 & 0,4 & & \\
\hline \multirow[t]{2}{*}{20} & \multirow{2}{*}{ Nado sincronizado } & Media & 5,2 & 4,8 & 1,3 & $-3,9$ & 3,1 \\
\hline & & DE & - & - & - & & \\
\hline \multirow[t]{2}{*}{21} & \multirow{2}{*}{ Patinaje artístico } & Media & 5,7 & 6,4 & 0,5 & $-5,1$ & 6,6 \\
\hline & & $\mathrm{DE}$ & - & - & - & & \\
\hline \multirow[t]{2}{*}{22} & \multirow{2}{*}{ Patín carrera } & Media & 3,4 & 2,9 & 2,0 & $-1,1$ & 1 \\
\hline & & DE & 1,0 & 3,2 & 0,7 & & \\
\hline \multirow[t]{2}{*}{23} & \multirow{2}{*}{ Salto ornamental } & Media & 3,4 & 4,4 & 2,2 & $-1,0$ & 2,9 \\
\hline & & $\mathrm{DE}$ & 0,7 & 0,8 & 0,9 & & \\
\hline \multirow[t]{2}{*}{24} & \multirow{2}{*}{ Taekwondo } & Media & 3,1 & 4,2 & 2,3 & $-0,7$ & 2,9 \\
\hline & & $\mathrm{DE}$ & 0,7 & 0,7 & 0,4 & & \\
\hline \multirow[t]{2}{*}{25} & \multirow{2}{*}{ Tenis de mesa } & Media & 4,6 & 4,4 & 1,9 & $-2,6$ & 2,1 \\
\hline & & $\mathrm{DE}$ & 0,7 & 0,7 & 0,8 & & \\
\hline \multirow[t]{2}{*}{26} & \multirow{2}{*}{ Tiro al arco } & Media & 5,7 & 5,1 & 1,1 & $-4,6$ & 3,3 \\
\hline & & DE & - & - & - & & \\
\hline \multirow[t]{2}{*}{27} & \multirow{2}{*}{ Tiro al vuelo } & Media & 5,5 & 4,2 & 2,3 & $-3,2$ & 0,5 \\
\hline & & $\mathrm{DE}$ & - & - & - & & \\
\hline \multirow[t]{2}{*}{28} & \multirow{2}{*}{ Voleyball playa } & Media & 4,9 & 4,3 & 1,8 & $-3,0$ & 1,9 \\
\hline & & DE & - & - & - & & \\
\hline & \multirow{2}{*}{ Total } & Media & 4,0 & 3,7 & 1,9 & $-2,1$ & 1,3 \\
\hline & & $\mathrm{DE}$ & 1,2 & 3,2 & 1,0 & & \\
\hline
\end{tabular}


en otros estudios llevados a cabo en deportistas de alto rendimiento $(3,4,22-25)$.

En voleibol indoor el somatotipo de los jugadores depende de la posición en la que juegue, por lo que no existe un consenso del somatotipo del jugador de voleibol, es por ello que se han reportado distintos somatotipos en dichos jugadores. En este estudio el somatotipo encontrado fue del tipo meso-ectomorfo, mismos somatotipo se encontró es

\section{FIGURA 3}

Somatocarta de los deportistas de sexo masculino de las disciplinas 1 a la 15 del Centro de Alto Rendimiento, Santiago de Chile.

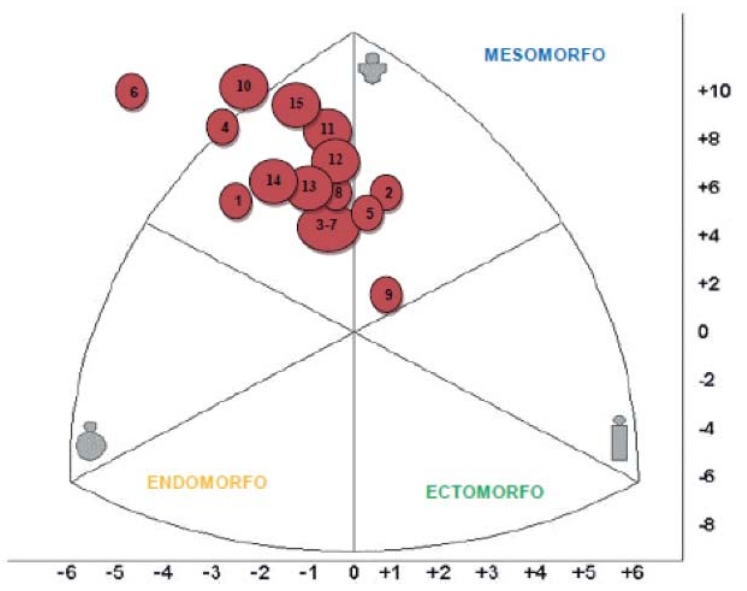

\begin{tabular}{|c|c|c|c|c|c|c|c|}
\hline $\mathbf{N}^{\circ}$ & Disciplina & & Endomorfo & Mesomorfo & Ectomorfo & $\mathbf{X}$ & $\mathbf{Y}$ \\
\hline \multirow[t]{2}{*}{1} & \multirow{2}{*}{ Andinismo } & Media & 8,1 & 6,2 & 1,6 & $-2,6$ & 5,9 \\
\hline & & DE & 9,7 & 1,1 & 1,1 & & \\
\hline \multirow[t]{2}{*}{2} & \multirow{2}{*}{$\begin{array}{l}\text { Atletismo carrera } \\
\text { medio fondo }\end{array}$} & Media & 2,0 & 4,0 & 3,8 & $-1,0$ & 6,3 \\
\hline & & DE & 0,5 & 0,8 & 0,4 & & \\
\hline \multirow[t]{2}{*}{3} & \multirow{2}{*}{$\begin{array}{l}\text { Atletismo carrera } \\
\text { velocidad }\end{array}$} & Media & 2,3 & 1,2 & 2,3 & 1,6 & 1,8 \\
\hline & & $\mathrm{DE}$ & 1,4 & 6,4 & 1,3 & & \\
\hline \multirow[t]{2}{*}{4} & \multirow{2}{*}{$\begin{array}{c}\text { Atletismo } \\
\text { lanzamiento bala }\end{array}$} & Media & 3,4 & 7,4 & 0,8 & $-0,6$ & 7,2 \\
\hline & & DE & - & - & - & & \\
\hline \multirow[t]{2}{*}{5} & \multirow{2}{*}{$\begin{array}{c}\text { Atletismo } \\
\text { lanzamiento jabalina }\end{array}$} & Media & 2,4 & 5,3 & 2,6 & $-3,1$ & 2,3 \\
\hline & & DE & - & - & - & & \\
\hline \multirow[t]{2}{*}{6} & \multirow{2}{*}{$\begin{array}{c}\text { Atletismo } \\
\text { lanzamiento martillo }\end{array}$} & Media & 6,4 & 9,4 & $-0,4$ & 2,0 & 1,8 \\
\hline & & DE & - & - & - & & \\
\hline \multirow[t]{2}{*}{7} & \multirow{2}{*}{ Atletismo salto largo } & Media & 1,6 & 5,6 & 2,7 & 0,5 & 2,4 \\
\hline & & $\mathrm{DE}$ & - & - & - & & \\
\hline \multirow[t]{2}{*}{8} & \multirow{2}{*}{ Bicicross } & Media & 3,3 & 5,9 & 1,9 & $-1,5$ & 7,0 \\
\hline & & DE & 1,3 & 1,5 & 1,2 & & \\
\hline \multirow[t]{2}{*}{9} & \multirow{2}{*}{ Bowling } & Media & 2,4 & 3,7 & 3,4 & $-1,5$ & 4,1 \\
\hline & & DE & 0,3 & 1,4 & 1,7 & & \\
\hline \multirow[t]{2}{*}{10} & \multirow{2}{*}{ Boxeo } & Media & 2,5 & 5,3 & 2,4 & $-0,8$ & 9,9 \\
\hline & & DE & 0,8 & 0,8 & 0,7 & & \\
\hline \multirow[t]{2}{*}{11} & \multirow{2}{*}{ Buceo } & Media & 2,1 & 6,7 & 1,3 & 0,2 & 5,7 \\
\hline & & DE & - & - & - & & \\
\hline \multirow[t]{2}{*}{12} & \multirow{2}{*}{ Canotaje } & Media & 2,3 & 5,7 & 2,1 & $-2,6$ & 10,6 \\
\hline & & $\mathrm{DE}$ & 0,7 & 1,2 & 0,8 & & \\
\hline \multirow[t]{2}{*}{13} & \multirow{2}{*}{ Ciclismo } & Media & 3,0 & 5,7 & 2,0 & $-0,7$ & 6,2 \\
\hline & & DE & 1,3 & 1,3 & 1,2 & & \\
\hline \multirow{2}{*}{14} & \multirow{2}{*}{ Esgrima } & Media & 3,6 & 6,2 & 1,3 & $-0,1$ & 5,5 \\
\hline & & DE & 0,8 & 0,5 & 0,5 & & \\
\hline \multirow[t]{2}{*}{15} & \multirow{2}{*}{ Esquí acuático } & Media & 2,8 & 3,0 & 7,4 & 0,9 & 7,4 \\
\hline & & $\mathrm{DE}$ & - & - & - & & \\
\hline
\end{tabular}


voleibolistas de India e Indonesia $(26,27)$. Sin embargo en jugadores profesionales de voleibol de Brasil y voleibolistas turcos el somatotipo encontrado fue mesomorfo balanceado $(11,28,29)$.
Deportistas de sexo femenino

Las deportistas de las disciplinas de andinismo, bowling, canotaje, lanzamiento de la jabalina, lanzamiento de disco, mountainbike, y salto ornamental se evidenció un somatotipo

\section{FIGURA 4}

Somatocarta de los deportistas de sexo masculino de las disciplinas 16 a la 33 del Centro de Alto Rendimiento, Santiago de Chile.

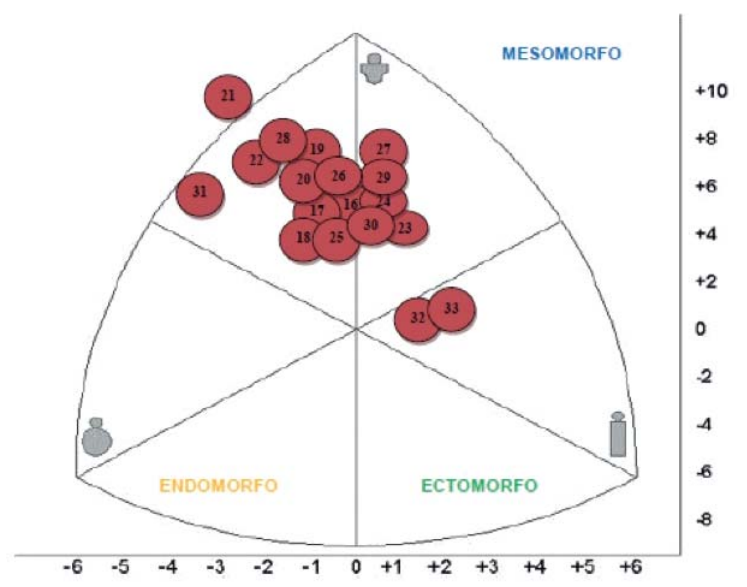

\begin{tabular}{|c|c|c|c|c|c|c|c|}
\hline \multirow[t]{2}{*}{16} & \multirow{2}{*}{ Gimnasia artística } & Media & 2,1 & 5,5 & 2,0 & $-0,2$ & 6,9 \\
\hline & & DE & 0,3 & 0,2 & 0,7 & & \\
\hline \multirow[t]{2}{*}{17} & \multirow{2}{*}{ Handball } & Media & 2,9 & 5,5 & 2,3 & $-0,6$ & 5,6 \\
\hline & & DE & 1,0 & 0,8 & 0,9 & & \\
\hline \multirow[t]{2}{*}{18} & \multirow{2}{*}{ Hockey patín } & Media & 3,5 & 4,8 & 1,8 & $-2,2$ & 7,4 \\
\hline & & $\mathrm{DE}$ & 1,0 & 1,6 & 0,7 & & \\
\hline \multirow[t]{2}{*}{19} & \multirow{2}{*}{ Judo } & Media & 2,6 & 3,2 & 1,5 & $-2,2$ & 10,9 \\
\hline & & DE & 0,3 & 7,3 & 0,9 & & \\
\hline \multirow[t]{2}{*}{20} & \multirow{2}{*}{ Karate } & Media & 3,0 & 5,8 & 1,5 & $-3,0$ & 8,8 \\
\hline & & $\mathrm{DE}$ & 1,0 & 0,6 & 0,4 & & \\
\hline \multirow[t]{2}{*}{21} & \multirow{2}{*}{ Levantamiento pesa } & Media & 3,3 & 5,8 & 0,8 & 0,01 & $-2,2$ \\
\hline & & $\mathrm{DE}$ & 1,6 & 5,0 & 1,2 & & \\
\hline \multirow[t]{2}{*}{22} & \multirow{2}{*}{ Lucha libre } & Media & 4,0 & 6,9 & 0,9 & 1 & 4,8 \\
\hline & & $\mathrm{DE}$ & 2,2 & 1,9 & 0,3 & & \\
\hline \multirow[t]{2}{*}{23} & \multirow{2}{*}{ Mountainbike } & Media & 1,7 & 4,7 & 2,8 & 1 & 1,8 \\
\hline & & $\mathrm{DE}$ & 0,2 & 0,4 & 0,6 & & \\
\hline \multirow[t]{2}{*}{24} & \multirow{2}{*}{ Natación } & Media & 2,2 & 3,7 & 2,7 & $-0,4$ & 6,8 \\
\hline & & $\mathrm{DE}$ & 0,5 & 3,6 & 0,7 & & \\
\hline \multirow[t]{2}{*}{25} & \multirow{2}{*}{ Patín carrera } & Media & 2,2 & 5 & 2,8 & $-0,4$ & 4,9 \\
\hline & & $\mathrm{DE}$ & 0,7 & 1,1 & 0,6 & & \\
\hline \multirow[t]{2}{*}{26} & \multirow{2}{*}{ Patinaje artístico } & Media & 2,3 & 5,6 & 1,6 & $-6,7$ & 12,8 \\
\hline & & $\mathrm{DE}$ & - & - & - & & \\
\hline \multirow[t]{2}{*}{27} & \multirow{2}{*}{ Remo } & Media & 1,5 & 5,7 & 2,1 & 1,8 & 2,1 \\
\hline & & $\mathrm{DE}$ & - & - & - & & \\
\hline \multirow[t]{2}{*}{28} & \multirow{2}{*}{ Rugby } & Media & 2,7 & 6,4 & 1,3 & $-1,4$ & 8,8 \\
\hline & & $\mathrm{DE}$ & 0,8 & 0,9 & 0,6 & & \\
\hline \multirow[t]{2}{*}{29} & \multirow{2}{*}{ Salto ornamental } & Media & 1,6 & 5,8 & 2,5 & 1 & 6,8 \\
\hline & & $\mathrm{DE}$ & - & - & - & & \\
\hline
\end{tabular}


predominantemente mesomorfo, lo que refleja deportistas con bajo porcentaje de masa grasa para su estatura, mayor masa muscular y desarrollo ósea para su estatura y una figura general en donde la linealidad de su cuerpo es relativamente baja. Las levantadoras de pesas y las karatecas de estudio también presentaron un somatotipo mesomórfico el cual coincide con el somatotipo encontrados en otros estudios $(11,30)$.

Las taewondokos chilenas al tener un somatotipo mesomorfo no se asemeja al perfil sugerido de las taewondoko de élite, el que corresponde a un somatotipo ecto-mesomórfico (24).

En el caso de las atletas de salto largo, corredoras de velocidad, bicicross, tiro al arco, tiro al vuelo, esgrimistas y las jugadoras de patín carrerea reportaron un somatotipo endomorfo, lo que se traduce en mayor adiposidad relativa, el cual es un factor perjudicial en su rendimiento deportivo a la hora de obtener mayores resultados $(9,14,17)$.

Situación similar ocurrió en las jugadoras de handball y la jugadora de voleyball playa en donde el somatotipo predominante fue el mesomórfico. Dicho somatotipo no es el ideal en la práctica de estos deportes en donde se ha reportado que las jugadoras de élite muestran valores superiores en peso corporal y masa libre de grasa, producto de un mayor porcentaje de masa muscular (31).

En disciplinas en donde la figura corporal es de gran importancia como ocurre en la gimnasia y patinaje artístico, las deportistas de élite de dichas disciplinas debiesen presentar un somatotipo meso-ectomórfico, sin embargo el somatotipo de las deportistas de este estudio es más bien endomorfo, lo cual representa mayor adiposidad relativa (32-34).

En el caso del nado sincronizado y el basquetball, las deportistas de ambas disciplinas en este estudio arrojaron un somatotipo más bien endomorfo, el cual defiere de las deportistas de elite de estos deportes, en donde el somatotipo reportado en estas atletas es del tipo central $(32,35)$.

Las atletas de carrera de medio fondo y valla presentaron un somatotipo del tipo central, el que corresponde a un equilibrio entre los tres componentes del somatotipo; sin embargo no pueden ser comparados dichos somatotipos ya que no existe una referencia internacional para las disciplinas en cuestión.

En el presente estudio reveló el somatotipo de los deportistas de élite chilenos tanto de sexo masculino como femenino, en donde la mayoría presentó un perfil mesomórfico, sin embargo existe un porcentaje de deportistas que no posee dicho somatotipo, sino más bien uno del tipo endomórfico, lo cual representa una desventaja a la hora de tener un óptimo rendimiento deportivo, debido al predominio de adiposidad corporal. Es por ello que todo programa de entrenamiento debe incluir rutinas de entrenamiento y una adecuada nutrición para lograr un mayor desarrollo de la masa musculo-esquelética del atleta, con el propósito de mejorar y optimizar su componente mesomórfico y, con ello incrementar el desempeño atlético, con el fin de lograr mayores logros deportivos a nivel internacional.

\section{CONCLUSIÓN}

En conclusión se describe el somatotipo de los deportistas pertenecientes a distintas disciplinas del Centro de Alto Rendimiento de Santiago de Chile, entregando información desde un punto de vista cineantropométrico al equipo multidisciplinario que trabaja con los distintos deportistas.

Los resultados de este estudio son de base para corregir las deficiencias actuales de los deportistas chilenos de alto rendimiento y además para completar la escasa información existente. También estos resultados son de ayuda para entrenadores, preparadores físicos, médicos, kinesiólogos y nutricionistas por nombrar algunos en la elaboración de planes de trabajo de captación, selección y formación de talentos, con la idea de contar con la mayor cantidad de deportistas de primer nivel.

Es por ello que es necesaria la elaboración de este tipo de estudios referentes con el somatotipo de nuestros deportistas, ya que aporta un punto de referencia para el equipo multidisciplinario que está detrás de estos atletas.

\section{RESUMEN}

El objetivo del estudio fue describir el somatotipo de los deportistas de distintas disciplinas del Centro de Alto Rendimiento de Santiago (CAR). Sujetos y métodos: Se evaluaron antropométricamente 309 deportistas de ambos sexos durante Marzo del 2010 hasta Abril del 2011. Características (promedio y D.E.): Mujeres: edad 19,6 \pm 4,8 años; peso, 62,1 $\pm 10,1 \mathrm{~kg}$; estatura, 162,7 $\pm 12,5 \mathrm{~cm}$; somatotipo, 4,0-3,7-1,9. Hombres: edad 21,1 \pm 5,9 años; peso, 78,8 \pm 11,6 kg; estatura, $176,5 \pm 8,3 \mathrm{~cm}$; somatotipo, 3,0-5,0-2,2. La mayoría de los deportistas de élite chilenos presentaron un perfil mesomórfi$\mathrm{co}$, sin embargo hay un porcentaje importante de deportistas que no obtuvo este somatotipo. Conclusiones: Este estudio podría servir de base para corregir las deficiencias actuales de los deportistas chilenos de alto rendimiento, siendo de ayuda para el equipo técnico en la elaboración de planes de trabajo de captación, selección y formación de talentos.

Palabras clave: somatotipo, deportistas, chilenos.

\section{BIBLIOGRAFÍA}

1. Rebato E, Rosique J. Estudio del somatotipo en la comarca de Busturia. Cuadernos de Antropología-Etnografía 1995; 12: 11-77.

2. Carter JEL. The Somatotypes of athletes - A review. Human Biol. 1970; 42: 535-69.

3. Pradas de la Fuente F, Carrasco L, Martínez E, Herrero R. Perfil antropométrico, somatotipo y composición corporal de jóvenes jugadores de tenis de mesa. Rev Int Cienc Deporte 2007; 7: 1123.

4. Carrasco L, Martínez E, Nadal C. Perfil antropométrico, somatotipo y composición corporal de jóvenes piragüistas. Rev Int Med Cienc Act Fís Deporte 2005; 20: 270-282.

5. Bagnall K, Kellett D. A study of potencial Olympic swimmers: I, the starting point. Brit J Sports Med 1977; 11(3): 127-32.

6. Ramírez E, Rivera J. Plan gallego de tecnificación deportiva: características morfológicas de sus nadadores. http://www. efdeportes.com 2006; 103.

7. Garay AL, Levine L, Carter JEL. Genetic and anthropological studies of olympic athletes. New York, Academic Press, 1974.

8. Luque A, Martínez A, López F, Martínez A, Villegas J. Valoración multimétodo de la composición corporal en karatecas. Arch Med Deporte 2006; 112: 109-15.

9. Pieter W, Bercades L. Somatotypes of combative sport athletes. Brazilian J Biomotr. 2009; 3 (1): 21-30.

10. Sterkowicz-Przybycień K.L. Body composition and somatotype of the top of polish male karate contestans. Biol Sports 2010; 27: 195-201.

11. Krawczyk B, Sklad M, Jackiewicz A. Heath-Carter somatotypes of athletes representing various sports. Biol Sports 1997; 14(4): 305-10. 
12. Irurtia $A$, Busquets $A$, Marina $M$, Galilea $P$, Carrasco $M$. Talla, peso, somatotipo y composición corporal en gimnastas de elite españoles desde la infancia hasta la edad adulta. Apunts Med Esport 2009; 161: 18- 28.

13. Betancourt H, Aréchiga J, Carvajal W. Estimación antropológica de la forma corporal de atletas elites cubanos de deportes olímpicos de combate. Antropo 2009; 19: 23-32.

14. Carrasco M, Irurtia A, Pons V, Iglesias X, Vidal E, Brotons $D$. Valoración nutricional de los hábitos alimentarios en jóvenes esgrimistas de competición. Apunts Medicina de l'Esport 2008; 159: 118-26.

15. Foley JP, Bird SR, White JA. Anthropometric comparison of cyclists from different events. Brit J Sports Med 1989; 23: 30-3.

16. Mathur DN, Toriola AL, Igbokwe NU. Somatotypes of nigerian athletes of several sports. Brit J Sports Med 1985; 19(4): 219-20.

17. Lozano R, Contreras D, Navarro L. Descripción antropométrica de los patinadores de velocidad sobre ruedas participantes en los Juegos Deportivos Nacionales de Venezuela. Rev Efdeportes. Año 11 - No 102 - Noviembre 2006.

18. Betancourt $H$, Sánchez G, Martínez M, Echevarría I. El somatotipo de Heath Carter en luchadores cubanos de alto rendimiento de los estilos libre y grecorromano. Rev Efdeportes. Año 8 - N 45 - Febrero de 2002.

19. Aamtya DL. Anthropometry physical fitness relative strength and heart rate study of nepalese weightlifters anthropometry physical fitness relative strength and heart rate response of nepalese weightlifters. http://dlamatyasports.com

20. Casagrande G, Viviani F. Somatotype of Italian rugby players. J Sports Med Phys Fitness 1993; 33(1): 65-9.

21. Rodríguez F, García S, Barraza F, Cabrera C, Siviero E. Variables antropométricas y su relación con el rendimiento físico en jugadores de rugby. Rev Efdeportes. Año $13-N^{\circ}$ 127 - Diciembre 2008

22. Yurian L, Gon F. Estudio de algunos indicadores de la selección de talentos en balonmanistas de la categoría 1314 años de ambos sexos en la EIDE. Revista Efdeportes. Año 10 - N 69 - Febrero de 2004.

23. Pelegrini A, Silva K. Perfil antropométrico e somatotipo de atletas da seleção brasileira de handebol junior participante do campeonato sulamericano. Rev Efdeportes. Año 11 N¹01 - Octubre de 2006.

24. Chan W, Pieter W, Moloney K. Kinathropometric profile of recreational taekwondo athletes. Biol Sport 2003; 20 (3): 175-79.

25. Muñoz C, Requena B, Zabala M. Determinación del perfil antropométrico de jóvenes corredores de medio fondo de élite. Rev Efdeportes. Año 8 - N 58 - Marzo de 2003.

26. Bandyopadhyay, A. Anthropometry and body composition in soccer and volleyball playerscin West Bengal, India. J Physiol Anthropol. 2007; 26: 501-505.

27. Rahmawati N, Budiharjo S, Ashizawa K. Somatotypes of young male athletes and non-athlete students in Yogyakarta, Indonesia. Anthropological Science 2007; 115: 1-7.

28. Andaki R, Andaki A, Mendes E. Perfil somatotípico de jugadores de voleibol profesional brasileño. Revista Efdeportes. Año 15 - No 145 - Junio de 2010.

29. Pelin C, Kürkçüoðlu A, Özener B, Canan A. Anthropometric Characteristics of Young Turkish Male Athletes. Coll Antropol. 2009; 33(4): 1057-63.

30. García O, Gutiérrez R. Descripción morfológica (masas segméntales, composición corporal y somatotipo) de la selección de levantamiento de pesas categoría femenina de Santander, Colombia. Rev Efdeportes. Año 12 - No 112 Septiembre de 2007.

31. García J, Cañadas M, Parejo I. Una revisión sobre la detección y selección de talento en balonmano. Rev Digital Deportiva e-balonmano.com 2007; 3(3): 39-46.

32. Betancourt H, Arechiga J, Ramirez CM. Análisis bioantropológico de la forma corporal de bailarinas de ballet y atletas de élite de deportes estéticos de Cuba. Rev Esp Antrop Fís 2009; 30: 75-82.

33. Díaz M, Mauri E, García Y, Jiménez C. Perfil antropométrico comparativo de la selección nacional de gimnasia artística femenina (2008) y el perfil del campeonato mundial en Rótterdam, Holanda (1987). Rev Efdeportes. Año $13-N^{\circ}$ 123 - Agosto de 2008.

34. Riffo C, Vallespir M, Barraza F. Caracterización del somatotipo y la composición corporal de gimnastas femeninas de nivel competitivo del Club Mabel González de Viña del Mar. Rev Efdeportes Año 15 - No 154 - Marzo de 2011. 